\title{
A method for nonlinear conjugate scales in a multi-position radar system with ambiguous range measurements
}

Volodymyr-Myron Miskiv, Ivan Prudyus, Piotr Kaniewski, Mateusz Pasternak

Volodymyr-Myron Miskiv, Ivan Prudyus, Piotr Kaniewski, Mateusz Pasternak, "A method for nonlinear conjugate scales in a multi-position radar system with ambiguous range measurements," Proc. SPIE 11442, Radioelectronic Systems Conference 2019, 114420C (11 February 2020); doi: $10.1117 / 12.2564088$ 


\title{
A method for nonlinear conjugate scales in a multi-position radar system with ambiguous range measurements
}

\author{
Volodymyr-Myron Miskiv ${ }^{*}$, Ivan Prudyus ${ }^{1 \dagger}$, Piotr Kaniewski ${ }^{2 \dagger}$, Mateusz Pasternak ${ }^{2 \S}$ \\ ${ }^{1}$ Lviv Polytechnic National University, 12 Bandery St., 79013 Lviv, Ukraine \\ ${ }^{2}$ Military University of Technology, Faculty of Electronics, Institute of Radioelectronics, \\ 2 Kaliski St., 00-908 Warsaw, Poland
}

\begin{abstract}
In this work, a two-dimensional analytical model of a multi-position radar system with ambiguous range measurements is elaborated and tested. In the proposed analytical model, properties of ambiguous measurements of fractional parts of relative unambiguity intervals in each radar are determined. Theorems are formulated defining the conditions of the unambiguous mapping of a target's coordinates onto the aforementioned fractional parts, as well as a reverse unambiguous mapping of those fractional parts onto a two-dimensional vector of integers. The vector contains ranges from the target to pairs of radars composing the considered system. The theorems are based on a principle of mapping the measurements of fractional parts onto a multidimensional unit cube, and the interpretation of the total set of measurements as a multilayer structure of this cube. Moreover, each layer is a multidimensional hypersurface bounded by the cube faces, and the unambiguity conditions are reduced to the conditions that these layers do not intersect with each other. Based on the developed model and the formulated theorems, an algorithm is proposed for disclosing the ambiguities of the fractional parts mentioned, as well as for obtaining unambiguous estimates of the target coordinates. An example of a multi-position radar system and results of modeling chosen elements of the algorithm for disclosing ambiguities are also presented. The aims of further research are formulated, particularly regarding the synthesis of multiposition radar systems and the elaboration of an analytical model for systems for the localization of emission sources of periodic radio signals.
\end{abstract}

Keywords: multi-position radar system, measurement unambiguity.

\section{INTRODUCTION}

Trends in modern radioelectronic system (RES) development have resulted in increasingly complex and often contradictory requirements for these systems. Concerning radar systems, such a contradiction consists of the expectation of extended range and improved effectiveness while at the same moment ensuring noise immunity and stealth operation. Such expectations, as a rule, cause an inconsistency in the requirements with respect to the desired parameters of the emitted radio signals and achievable ranges of the RES. There are well-known approaches to partially solve the problems stated above. They consist of using radio signals with complex types of modulation [1-6], or combining single measurements from different radars [1], [7-8] in order to obtain a desired effect in a multi-position radar system (MPRS).

In this paper, an approach to solve the above contradiction is proposed. It involves using modular code binary sequences (CBSs) in radars with structures optimized by the criteria of secrecy and noise immunity [1], [4-6], but with ambiguous estimation of the radar-target distance. Additionally, the approach involves the joint processing of ambiguous measurements of each of the radars, and on this basis, getting unambiguous estimates of the target coordinates. The implementation of the proposed approach is based on the construction of a two-dimensional MPRS analytical model, using a method of nonlinear conjugation of ambiguous scales and an algorithm of ambiguity disclosure.

\footnotetext{
*mmiskiv@1p.edu.ua

† iprudyus@polynet.lviv.ua

* piotr.kaniewski@wat.edu.pl

$\S$ mateusz.pasternak@wat.edu.pl
}

Radioelectronic Systems Conference 2019, edited by Piotr Kaniewski, Jan Matuszewski, Proc. of SPIE Vol. 11442, 114420C · () 2020 SPIE · CCC code: 0277-786X/20/\$21 · doi: 10.1117/12.2564088 


\section{PROBLEM FORMULATION}

It is assumed that each radar operates in an active mode, emitting a continuous radio signal modulated by the optimal CBS. In general, modulators of the CBS are paired differently and are quasi-orthogonal for each pair of radars. Thus, all radars emit radio signals with quasi-orthogonal modulations, which ensures their effective identification when receiving and processing reflected radio targets. The duration of one cycle of a modulated radio signal (repetition period $T_{i}$ of the modulating CBS in the radio signal of the $i$-th radar) should not exceed the value $R_{\min } / c$, where $R_{\text {min }}$ represents the minimum distance to the target; $c$ is the free-space electromagnetic wave velocity.

The interval (scaled in distance units) of a single distance measurement of the radar is determined by the ratio $d_{i}=T_{i} \cdot c$. The time of the radio signal passing from the radar to the target and back is $\mathrm{T}_{\text {pass }}=2 R_{i} / c$, where $R_{\mathrm{i}}$ is the distance, and $R_{i} / d_{i}=\mathrm{T}_{\text {pass }} \cdot c /\left(2 d_{i}\right)$ is the relative distance from the $i$-th radar to the target. The receiver of the $i$-th radar estimates the fractional part of the relative distance $R_{i} / d_{i}$. The corresponding ratios can be written as follows:

$$
\left[R_{i} / d_{i}\right]^{+}+\left\{R_{i} / d_{i}\right\}^{+}=T_{\text {pass }} / 2 T_{i}
$$

where []$^{+}$is a floor function of $R_{i} / d_{i}$ and \{\}$^{+}$means a fractional part function of $R_{i} / d_{i}$;

$\left[R_{i} / d_{i}\right]^{+}=K_{i}$ - unknown number of entire relative periods in the distance $R_{i} / d_{i}$;

$\left\{R_{i} / d_{i}\right\}^{+}=\phi_{i}$ - fractional part of the relative period at the distance $R_{i} / d_{i}$ for $i \in\{1,2, \ldots, n\}$.

From (1) the following relations between the relative distance, period $T_{i}$ and time interval $\Delta t_{i}$ follow, which define the fractional part $\phi_{i}$ :

$$
\left\{\mathrm{T}_{\text {pass }} /\left(2 \mathrm{~T}_{\mathrm{i}}\right)\right\}^{+}=\left\{\Delta \mathrm{t}_{\mathrm{i}} / \mathrm{T}_{\mathrm{i}}\right\}^{+}=\mathrm{f}_{\mathrm{i}}, \quad\left\{2 R_{i} / d_{i}\right\}^{+} \cdot T_{i}=\left\{2 \phi_{i}\right\}^{+} \cdot T_{i}=\Delta t_{i}
$$

Thus, the time interval $\Delta t_{i}$, which can be estimated in the $i$-th radar using the reflected radio signal, is determined by relation (2). In order to further formulate the MPRS model, we will need to establish an analytic formula that expresses the value of $\phi_{i}$ by means of $\left\{2 \phi_{i}\right\}^{+} \cdot T_{i}$. From expression (2), we can write the following congruence:

$$
\left\{2 \phi_{i}\right\}^{+}=\Delta t_{i} / T_{i} ; \quad T_{i}>0, \quad 0 \leq \Delta t_{i}<T_{i}, \quad 0 \leq \phi_{i}<1 .
$$

Formula (3) has the following solution:

$$
\phi_{i 1}=\Delta t_{i} /\left(2 T_{i}\right) ; \phi_{i 2}=\Delta t_{i} /\left(2 T_{i}\right)+0.5
$$

The plot of $\phi_{i}$ vs $\Delta t_{i} / T_{i}$ is shown in Fig. 1 .

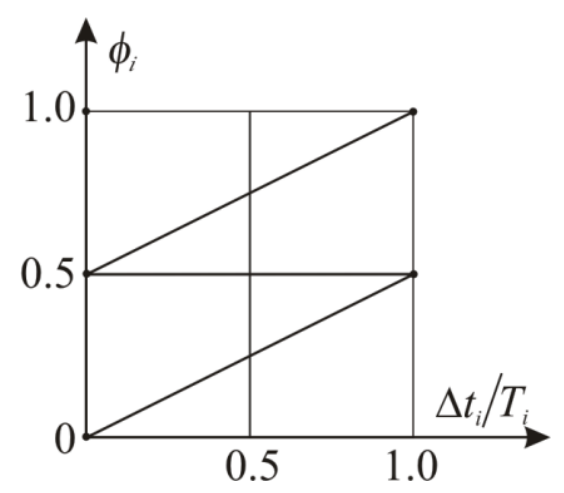

Figure 1. Dependence of $\phi_{i}$ value on $\Delta t_{i} / T_{i}$ 
Equation (4) and Fig. 1 imply that $\Delta t_{i} / T_{i}$ is mapped onto $\phi_{i}$ ambiguously.

The schematic representation shown in Fig. 2 is used to form an analytical two-dimensional model of the MPRS.

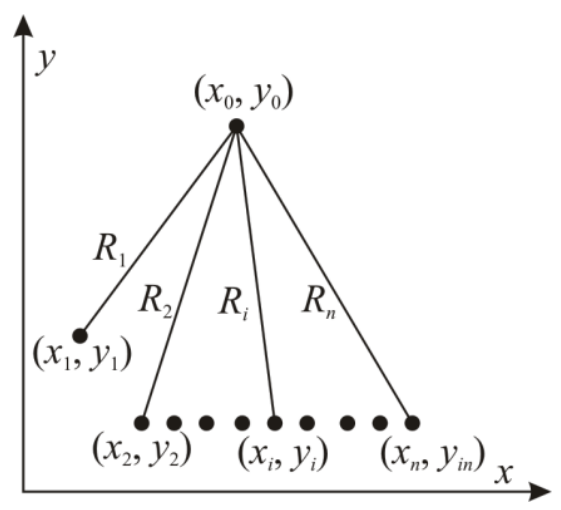

Figure 2. Two-dimensional representation of MPRS

From the above, it follows that a separate $i$-th radar can estimate a fractional part of $\phi_{i}$ but cannot determine the whole value $K_{i}$. Thus, the problem can be solved by developing a deterministic analytical model of the MPRS and determining the conditions for unambiguous mapping of coordinates $x_{0}, y_{0}$ onto multiple values $\phi_{i}$. In addition, it is necessary to determine the conditions for the unambiguous map of values $\phi_{i}$ in $K_{i}$ for two selected radars to elaborate an algorithm for the disclosure of an ambiguity. Note that the term "deterministic model" used here means that the values $\phi_{i}$ are not random.

To solve this problem, the approach developed in [9-11] concerning the methods of revealing ambiguity in the linear conjugation of scales was developed.

\section{ANALYTICAL MODEL OF A MULTI-POSITION RADAR SYSTEM}

From the above considerations, a deterministic analytical model of the radar can be represented in the form of a system of nonlinear congruences:

$$
\left\{\sqrt{\left(x_{0}-x_{i}\right)^{2}+\left(y_{0}-y_{i}\right)^{2}} / d_{i}\right\}^{+}=\phi_{i}
$$

where $x_{0}, y_{0}$ - target coordinates; $x_{i}, y_{i}$ - coordinates of the $i$-th radar. It allows the two following statements to be formulated:

Statement 1: a necessary and sufficient condition for unambiguity mapping $\left(x_{0}, y_{0}\right) \rightarrow \phi_{i}$ in the system of nonlinear congruences (5) has the form of inequality: $\sqrt{\left(x_{0}-x_{i}\right)^{2}+\left(y_{0}-y_{i}\right)^{2}} / d_{i}>0$.

For two arbitrarily selected $i$-th and $j$-th radars, a system of equations can be obtained from (5) by elementary transformations:

$$
\sqrt{\left(x_{0}-x_{i}\right)^{2}+\left(y_{0}-y_{i}\right)^{2}}=K_{i} d_{i}+\Delta R_{0 i}, \quad \sqrt{\left(x_{0}-x_{j}\right)^{2}+\left(y_{0}-y_{j}\right)^{2}}=K_{j} d_{j}+\Delta R_{0 j},
$$

where $\Delta R_{0 i}=\phi_{i} d_{i}, \Delta R_{0 j}=\phi_{j} d_{j}$. 
In general, from (5) we can write $n$ independent equations similar in form to (6). The values $K_{i}$ and $K_{j}$ are unknown integer parameters, $x_{0}, y_{0}$ are unknown coordinates of the target, and $\Delta R_{0 i}, \Delta R_{0 j}$ are known values. Therefore, it is necessary to express $x_{0}, y_{0}$ from (6) by $K_{i} \cdot d_{i}, K_{j} \cdot d_{j}$ and $\Delta R_{0 i}, \Delta R_{0 j}$. Next, substitute $x_{0}, y_{0}$ to the rest of $n-2$ equations that are formed from (5) and obtain $n-2$ equations that contain $n$ unknown integer values $K_{m}$ for $m \in\{1, \ldots, n\}, m \neq i, j$. An essential feature of the obtained integer equations is that the integer parameters $K_{m}$ enter linearly into equations with indices $m$. Next, for the resulting system of $n-2$ equations with $n$ unknown integer variables $K_{m}$, conditions under which this system has a single solution are formulated.

Therefore, based on this consideration, when carrying out the equivalent transformations of the system (6), the following expressions for $x_{0 i}, y_{0 i}$ can be obtained:

$$
\begin{aligned}
& x_{0 i}=0.5 \cdot x_{i j}\left(A_{i}^{2}-A_{j}^{2}-R_{j i}^{2}\right) / R_{j i}^{2} \pm 0.5 \cdot y_{i j} \sqrt{2\left(A_{i}^{2} A_{j}^{2}+A_{i}^{2} R_{j i}^{2}+A_{j}^{2} R_{j i}^{2}\right)-A_{i}^{4}-A_{j}^{4}-R_{j i}^{4}} / R_{j i}^{2} \\
& y_{0 i}=0.5 \cdot y_{i j}\left(A_{i}^{2}-A_{j}^{2}-R_{j i}^{2}\right) / R_{j i}^{2} \mp 0.5 \cdot x_{i j} \sqrt{2\left(A_{i}^{2} A_{j}^{2}+A_{i}^{2} R_{j i}^{2}+A_{j}^{2} R_{j i}^{2}\right)-A_{i}^{4}-A_{j}^{4}-R_{j i}^{4}} / R_{j i}^{2}
\end{aligned}
$$

where $A_{i}=K_{i} \cdot d_{i}+\Delta R_{0 i} ; A_{j}=K_{j} \cdot d_{j}+\Delta R_{0 j} ; x_{i j}=x_{i}-x_{j} ; \quad y_{i j}=y_{i}-y_{j} ; R_{i j}^{2}=x_{i j}^{2}+y_{i j}^{2} ; x_{0 i}=x_{0}-x_{i} ; \quad y_{0 i}=y_{0}-y_{i}$.

Substituting (7) and (8) into (6) with index $m \neq i, j$ and carrying out a series of transformations gives the following equation with three unknown integers $K_{i}, K_{j}, K_{m}$ :

$$
\begin{gathered}
\left(x_{i j} x_{i m}+y_{i j} y_{i m}\right)\left(A_{i}^{2}-A_{j}^{2}-R_{j i}^{2}\right) / R_{j i}^{2} \pm \\
\pm\left(y_{i j} x_{i m}-x_{i j} y_{i m}\right) \sqrt{2\left(A_{i}^{2} A_{j}^{2}+A_{i}^{2} \cdot R_{j i}^{2}+A_{j}^{2} \cdot R_{j i}^{2}\right)-A_{i}^{4}-A_{j}^{4}-R_{j i}^{4}} / R_{j i}^{2}= \\
=\left(K_{m} \cdot d_{m}+\Delta R_{0 m}\right)^{2}-\left(K_{i} \cdot d_{i}+\Delta R_{0 i}\right)^{2}-R_{i m}^{2}
\end{gathered}
$$

The practical significance has the variant of MPRS radar placement on a straight line which is taken as the $x$-axis of the coordinate system (Fig. 2). In this case, the values $y_{i j}$ for $i \neq j, i \in\{1, \ldots, n\}$ are equal zeroes $y_{i j}=0$, and (9) can be written after a series of transformations in the following form:

$$
\left[\left(K_{j}+\phi_{j}\right)^{2} \cdot d_{j}^{2} x_{m i}-\left(K_{i}+\phi_{i}\right)^{2} \cdot d_{i}^{2} x_{m j}\right] / d_{m}^{2} x_{j i}+x_{m i} \cdot x_{m j} / d_{m}^{2}=\left(K_{m}+\phi_{m}\right)^{2} ; i \neq j ; m \neq i, j
$$

The equation (10) with integer variables $K_{i}, K_{j}, K_{m}$ for $i \neq j$ and $m \neq i, j$ provides the ability to write down the system of $n-2$ nonlinear congruences, each containing only two integer variables $K_{i}$ and $K_{j}$ :

$$
\left\{\sqrt{\left[\left(K_{j}+\phi_{j}\right)^{2} \cdot d_{j}^{2} x_{m i}-\left(K_{i}+\phi_{i}\right)^{2} \cdot d_{i}^{2} x_{m j}\right] / d_{m}^{2} x_{j i}+x_{m i} x_{m j} / d_{m}^{2}}\right\}^{+}=\phi_{n} .
$$

The system of congruences (11) gives the following mapping: for each fixed pair of values $K_{i}, K_{j}$ selected from the set of integers, the set of left-hand sides of the congruence system (11) defines $\left\{\left(\phi_{i}, \phi_{j}\right) \rightarrow\left(\phi_{1}, \phi_{2}, \ldots, \phi_{m}, \ldots, \phi_{m}\right)\right\}$ for $i \neq j$ and $m \neq i, j$. This map is continuous and sets the $n-2$ dimensional surface in a single cube, and provides a tool for forming multiple values $K_{i}, K_{j}$ within which unambiguous mapping $\left(x_{0}, y_{0}\right) \Leftrightarrow\left(\phi_{1}, \phi_{2}, \ldots, \phi_{n}\right) \Leftrightarrow\left(K_{i}, K_{j}\right)$ is ensured.

Statement 2: a necessary and sufficient condition for mutual unambiguous mapping in the form $\left(x_{0}, y_{0}\right) \Leftrightarrow\left(\phi_{1}, \phi_{2}, \ldots, \phi_{n}\right) \Leftrightarrow\left(K_{i}, K_{j}\right)$ requires that there is no congruence solution:

$$
\left\{\left[x_{m i}\left(K_{j p}^{2}-K_{j q}^{2}\right)-2 \phi_{j} x_{m i}\left(K_{j p}^{2}-K_{j q}^{2}\right)\right] / d_{j}^{2}-\left[x_{m i}\left(K_{i r}^{2}-K_{i l}^{2}\right)-2 \phi_{i} x_{m j}\left(K_{i r}^{2}-K_{i l}^{2}\right)\right] / d_{i}^{2}\right\}^{2}=0
$$


where $\phi_{i}, \phi_{j} \in[0 \div 1), \quad K_{j p}, K_{j q} \in \Theta_{j}, \quad K_{i r}, K_{i l} \in \Theta_{i}$ and $\Theta_{i}, \Theta_{j}$ are sets of $K_{i}, K_{j}$ values within the range of unambiguity.

The system of congruences (11) gives a practical algorithm for obtaining estimates of $K_{i}, K_{j}$ values and calculating the coordinates by the equations (7) and (8).

The sequence of the algorithm steps is as follows:

1. The known input values in (11) are $\phi_{i}, \phi_{j}, \phi_{m}, d_{i}, d_{j}, x_{j i}, x_{m i}, x_{m j}$ and $\Theta_{i j}$ set of values $\left(K_{i}, K_{j}\right)$.

2. Calculate the value of the left-hand sides of (11), substituting in turn their values $\left(K_{i}, K_{j}\right)$ from the set of $\Theta_{i} \times \Theta_{j}$. For each $\left(K_{i}, K_{j}\right)$ can be received $n-2$ values of the left parts, and there will be $M \cdot(n-2)$ of them in total, where $M$ is the number of elements of the set $\Theta_{i} \times \Theta_{j}$.

3. Evaluation $\left(K_{i}, K_{j}\right)$, which determines the coordinates $\left(x_{0}, y_{0}\right)$ is obtained by the following criterion:

$$
\min _{K_{i}, K_{j} \in \Theta_{i} \times \Theta_{j}} \sum_{m \neq i, j ; m \in\{1 ; 2 ; \ldots, n\}}\left|\xi_{i j m}-\phi_{m}\right| .
$$

where $\xi_{i j m}$ represents the left-hand sides of (11).

\section{NUMERICAL EXAMPLE}

A quantitative example for a two-dimensional radar system is shown below.

$$
\begin{aligned}
& \left(x_{0}, y_{0}\right)=(7 ; 23), \mathrm{R}_{1} / \mathrm{d}_{1}=3.4345185, \mathrm{R}_{2} / \mathrm{d}_{2}=4.07074408 \\
& \left(x_{1}, y_{1}\right)=(0 ; 0),\left(x_{2}, y_{2}\right)=(2 ; 0),\left(x_{3}, y_{3}\right)=(5 ; 0) \\
& d_{1}=7.0, d_{2}=5.0, d_{3}=3.0 \\
& \phi_{1}=0.4345185, \phi_{2}=0.7074408, \phi_{3}=0.6955973 .
\end{aligned}
$$

A set of permissible values of $\left(K_{i}, K_{j}\right)$ is shown in Table 1 .

Table 1. The set of permissible pairs $\left(K_{1}, K_{2}\right)$

\begin{tabular}{|c|c|c|c|c|c|c|c|c|c|}
\hline$K_{2}$ & 0 & 1 & 2 & 3 & 4 & 5 & 6 & 7 & 8 \\
\hline 0 & + & + & + & + & + & + & + & + & + \\
\hline 1 & & + & + & + & + & + & + & + & + \\
\hline 2 & & & + & + & + & + & + & + & + \\
\hline 3 & & & & + & + & + & + & + & + \\
\hline 4 & & & & & & + & + & + & + \\
\hline 5 & & & & & & & + & + & + \\
\hline 6 & & & & & & & & + & + \\
\hline
\end{tabular}

The results of the calculations according to criterion (13) are shown in Table 2. 
Table 2. Results of $\left(K_{1}, K_{2}\right)$ calculations using criterion (13)

\begin{tabular}{|c|c|c|c|c|c|c|c|c|c|}
\hline$K_{\mathbf{2}} K_{\mathbf{1}}$ & 0 & 1 & 2 & 3 & $\mathbf{4}$ & 5 & 6 & 7 & 8 \\
\hline 0 & 0.5786 & 0.0858 & 0.4909 & 0.1254 & 0.2503 & 0.6222 & 0.079 & 0.3600 & 0.2717 \\
\hline 1 & & 0.1395 & 0.0670 & 0.2135 & 0.5160 & 0.1592 & 0.1778 & 0.5221 & 0.1288 \\
\hline 2 & & & 0.0543 & 0.0433 & 0.0852 & 0.3038 & 0.5687 & 0.1390 & 0.1697 \\
\hline $\mathbf{3}$ & & & & 0.2238 & $\mathbf{0 . 0 0 6 3}$ & 0.0366 & 0.1796 & 0.3856 & 0.6307 \\
\hline 4 & & & & & & 0.0845 & 0.0352 & 0.1121 & 0.2636 \\
\hline 5 & & & & & & & 0.1798 & 0.0659 & 0.0852 \\
\hline 6 & & & & & & & & 0.2864 & 0.1189 \\
\hline
\end{tabular}

The calculations performed according to the developed algorithm restore the values $\left(K_{i}, K_{j}\right)$, which unambiguously, by the formulae (7) and (8), provide the determination of coordinates $\left(x_{0}, y_{0}\right)$.

\section{CONCLUSIONS}

The paper presented a two-dimensional analytical model of a multi-position radar system with ambiguous measurements of distances from each of the radars of the system, a method of the nonlinear conjugation of radar measuring scales, and an algorithm for revealing the ambiguity of measurements of a set of radar scales. The developed algorithm provides an effective ambiguity disclosure since the dimension of the recoverable two-dimensional parameter does not depend on the number of radars in the system. An example of a two-dimensional multi-position radar system and the results of a numerical simulation of the ambiguity detection algorithm elements were given.

From the studies and the results obtained in this work, the following directions of designing multi-position radar systems can be formulated:

- further development of an analytical MPRS model for the three-dimensional case with consideration of the stochastic nature of $\phi_{m}$ in (5),

- development of an analytical model and an effective method of detecting ambiguity in passive systems for determining the coordinates of radiation sources of periodic radio signals,

- synthesis of MPRS scales with the active radar mode,

- synthesis of scales of passive systems for determining coordinates of periodic signals of radiation sources.

The obtained results provide a good basis for enabling the implementation of multi-position radar systems.

\section{REFERENCES}

[1] Skolnik M.I., "Radar Handbook," 3rd Ed. NewYork: McGraw-Hill (2008).

[2] Levanon N. and Mozeson E., "Radar Signals," New York: IEEE Press, John Wiley \&Sons, Inc. (2004).

[3] Cook C.E. and Bernfield M., "Radar Signals: An Introduction to Theory and Application," New York: Academic Press (1967).

[4] Packebusch T., Mertens S., "Low Autocorrelation Binary Sequences," Journal of Physics A Mathematical and Theoretical 49 (16) (2016).

[5] Miskiv V.M., Prudyus I.N. and Yankevych R.V., "Optimization of Code Constructions of Binary Sequences Class on a Basis of Set Theoretical Representation," Radioelectronics and Communications Systems, Volume 61, Number 7, pp. 292-305 (2018). 
[6] Miskiv V.M., Kaniewski P., Prudyus I., and Konatowski S., "Method of synthesis of signal-code constructions for secretive noise-proof data transmission radio systems," Proc. of SPIE, Volume 11055, Art. No. 1105516 (2019).

[7] Salah J.E. and Morriello J.E., "Development of a multistatic measurement system," IEEE International Radar Conference, pp. 88-93 (1980).

[8] Willis N.J. and Griffiths H.D., (eds.), “Advanced in Bistatic Radar, Raleigh,”, NC: Sci Tech Publishing Inc. (2007).

[9] Skrypnik G.I., "On the recurrent procedure of disclosing ambiguity of phase measurements," Autometry, Novosibirsk, No. 3, pp. 8-13 (1978), https://www.iae.nsk.su>articles-archive (in Russian).

[10] Miskiv M.V. and Skrypnik G.I., "On the choice of the number of scales and ratios of scales in a multiscale measuring system," Autometry, Novosibirsk, No. 4, pp. $93-98$ (1979), https://www.iae.nsk.su>articles-archive (in Russian).

[11] Popov Yu.D., "On a problem of optimal choice of a decision rule for an alphabetical scheme for eliminating the ambiguity of cyclic measurements," Autometry, Novosibirsk, No. 3, pp. 7-8 (1978), https://www.iae.nsk.su>articles-archive (in Russian). 